\title{
BIBLIOGRAFÍA IBEROAMERICANA DE DERECHO PENAL Y CRIMINOLOGÍA
} (Abril-Septiembre de 2001)

\section{ARGENTINA}

(Corresponsales: D. Gustavo Eduardo Aboso, D. Sandro Fabio Abraldes y D. Javier de la Fuente)

\section{A) Libros}

Aвoso, Gustavo Eduardo, y Abraldes, Sandro F.: Digesto Práctico La Ley, Excarcelación, La Ley, Buenos Aires, 2001.

Авоsо, Gustavo Eduardo: El delito de defraudación por administración infiel, B de F, Buenos Aires, 2001.

Agüero, Arístides: Digesto contravencional, Dike, Bs. As., 2001.

Arnaudo, Luis (comp.): Fallos de la Casación Penal, Di Plácido, Bs. As., 2001.

Aued, Norberto Rubén: Probation, Universidad, Bs. As., 2001.

Bacigalupo, Silvina: Responsabilidad penal de las personas jurídicas, Hammurabi, Bs. As., 2001.

Bouzat, Andrés; Esand, Luis, y Navarro, Pablo: Juez y ley penal: un análisis de la interpretación y aplicación de las normas penales, Alveroni, Bs. As., 2001.

Breglia Arias, Omar: Código Penal y Leyes Complementarias, Astrea, Bs. As., 2001.

Bunge Campos, Luis (comp.): Las garantías penales y procesales: un enfoque histórico comparado, Del Puerto, Bs. As., 2001.

CAPolupo, Enrique: Ladrones de inocencia, Abuso, pedofilia, criminalidad de los cuellos verdes. La explotación sexual comercial de los niños, Campomanes, Buenos Aires, 2001.

Cerezo Mir, José: Temas Fundamentales del Derecho Penal, Rubinzal-Culzoni, Bs. As., 2001.

Clemente, José: Usurpación, Marcos Lerner, Córdoba, 2001. 
Crimen y Castigo, Cuaderno del Departamento de Derecho Penal y Criminología de la Facultad de Derecho, Universidad Nacional de Buenos Aires, año 1, agosto 2001, Nro. 1.

DaYenoff, David Elvio: Delitos. Una visión práctica, Depalma, Bs. As., 2001.

Donna, Edgardo A.: Derecho Penal Parte Especial, Tomos II-A y II-B, Rubinzal-Culzoni, Bs. As., 2001.

Figari, Rubén E.: Hurtos simples agravados, Jurídicas Cuyo, Mendoza, 2001.

Fleita, Benito: Sistemas actuales de análisis en criminalística, La Rocca, Bs. As., 2001.

García Elorrio, Aurelio: Protección de los niños no nacidos en el sistema interamericano de derechos humanos, Advocatus, Bs. As., 2001.

García Vitor, Enrique: Régimen legal de los estupefacientes, Jurídicas Cuyo, Mendoza, 2001.

Guemureman, Silvia: La Niñez ajusticiada, Ed. Del Puerto, Bs. As., 2001.

Laje Anaya, Justo: Estudios de derecho penal, Marcos Lerner, Córdoba, 2001.

Messuti, Ana: El tiempo como pena, Campomanes, Buenos Aires, 2001.

Muñoz Conde, Francisco: Introducción al derecho penal, en colección «Maestros del Derecho Penal», Vol. 3, Gonzalo D. Fernández (Director), Gustavo E. Aboso (Coordinador), B de F, Buenos Aires, 2001.

Nuevas Formulaciones en las ciencias penales, Homenaje a Claus Roxin, Facultad de Derecho y Ciencias Sociales de la Universidad Nacional de Córdoba, (Ed. La Lectura y Lerner), Córdoba, 2001.

Parma, Carlos: Pensamiento de Günther Jakobs. El Derecho Penal del siglo XXI, Jurídicas Cuyo, Mendoza, 2001.

Política criminal, derechos humanos y sistemas en el siglo XXI: homenaje al Dr. Pedro Rubens David, Ed. Depalma, Bs. As., 2001.

Righi, Esteban: Teoría de la pena, Hammurabi, Bs. As., 2001.

Rocco, Arturo: El objeto del delito y de la tutela jurídica penal, en colección «Maestros del Derecho Penal», Vol. 2, Gonzalo D. Fernández (Director), Gustavo E. Aboso (Coordinador), B de F, Buenos Aires, 2001.

Rodríguez, Agustín W.: Delitos contra la propiedad, Juris, Bs. As., 2001.

Rodríguez, Agustín: Fundamentos de derecho penal y criminología, Juris, Bs. As., 2001.

Ruiz, Alicia E.: Idas y vueltas. Por una teoría crítica del derecho, Del Puerto, Bs. As., 2001.

Rusconi, Maximiliano (compilador): Delitos contra la administración de justicia, Ciudad Argentina, Bs. As., 2001.

Rusconi, Maximiliano: La justificación en el derecho penal, 2da. Ed. Ad Hoc., Bs. As., 2001.

SARrulle, Oscar: Dogmática de la culpabilidad, Universidad, Bs. As., 2001. 
SLOKAR, A., y VIRGOLINI, J.: Nada personal: estudios críticos sobre la cuestión criminal, Depalma, Bs. As., 2001.

Sproviero, Juan H.: Prescripción de la acción y de la pena, Abaco, Bs. As., 2001.

TENCA, Adrián M.: Delitos sexuales, Astrea, Bs. As., 2001.

Terradillo Basoco, Juan: Empresa y derecho penal, Ad-Hoc, Bs. As., 2001.

Vergara LuQue, José A.: Imputabilidad e inimputabilidad penal, Jurídicas Cuyo, Mendoza, 2001.

Welzel, Hans: El nuevo sistema del derecho penal, Una introducción a la doctrina de la acción finalista, trad. del Prof. Dr. José Cerezo Mir, en colección «Maestros del Derecho Penal», Vol. 4, Gonzalo D. Fernández (Director), Gustavo E. Aboso (Coordinador), B de F, Buenos Aires, 2001.

Young, Jock: ¿Qué hacer con la ley y el orden?, trad. Por Mariano Ciafardini, Del Puerto, Bs. As., 2001.

\section{B) Artículos}

Aвoso, Gustavo E.: El nuevo Código Procesal Penal francés y el afianzamiento de las garantías de las personas sometidas a proceso (A propósito del affaire «Selmouni» resuelto por la Corte Europea de Derechos Humanos), Suplemento Actualidad, La Ley, I y II Parte, del 18 y 23 de octubre de 2001.

Aвoso, Gustavo E., y Donna, Edgardo A.: Obligaciones alimentarias de los padres y el delito de incumplimiento de los deberes de asistencia familiar (art. 1. ${ }^{\circ}$ de la ley 13.944), en Revista de Derecho Privado y Comunitario, Alimentos, 2001-1, Rubinzal Culzoni, Santa Fé, 2001, pp. 247 y ss.

Aвоso, Gustavo E.: Defraudación por administración infiel, autoría mediata y delito especial, Doctrina Judicial, Año XVII, N. ${ }^{\circ} 46$, del 14 de noviembre de 2001, pp. 717 y ss.

Bargalló, Martín, y Ranuschio, Daniel C.: Pena de prisión y sistemas alternativos, Jurisprudencia Argentina, N. ${ }^{\circ}$ 6245, Buenos Aires, 9 de mayo de 2001, pp. 9 y ss.

Bertoni, Eduardo A.: La tensión entre la libertad de expresión y el derecho penal, Suplemento de Jurisprudencia Penal, La Ley, Buenos Aires, 7 de septiembre de 2001, pág. 19 y siguientes.

Boldova Pasamar, Miguel Angel: Tenencia y consumo de drogas: los límites de las prohibiciones en el Derecho español. Revista de Derecho Penal, Nro. 2001-1, Ed. Rubinzal-Culzoni, Bs. As., 2001.

Boumpadre, Jorge Eduardo: La prevaricación judicial en el Código Penal argentino, en Nuevas Formulaciones en las ciencias penales, Homenaje a Claus Roxin, Facultad de Derecho y Ciencias Sociales de la Universidad Nacional de Córdoba (Ed. La Lectura y Lerner), Córdoba, 2001. 
BRUERA, Matilde: Autoría y dominio de la voluntad a través de aparatos organizados de poder, en Nuevas Formulaciones en las ciencias penales, Homenaje a Claus Roxin, Facultad de Derecho y Ciencias Sociales de la Universidad Nacional de Córdoba (Ed. La Lectura y Lerner), Córdoba, 2001.

Cafferata Nores, José I.: Garantías y sistema constitucional. Revista de Derecho Penal, Nro. 2001-1, Ed. Rubinzal-Culzoni, Bs. As., 2001.

«Caso Pinochet» (España, Reino Unido y Chile), Suplemento Especial de Derecho Constitucional, La Ley, Buenos Aires, 11 de septiembre de 2001, $150 \mathrm{pp}$.

Cesano, José Daniel: Reparación y resolución del conflicto penal: su tratamiento en el Código Penal argentino, en Nuevas Formulaciones en las ciencias penales, Homenaje a Claus Roxin, Facultad de Derecho y Ciencias Sociales de la Universidad Nacional de Córdoba (Ed. La Lectura y Lerner), Córdoba, 2001.

CóRDOBA, Fernando Jorge: La capacidad de motivación y la imputación de culpabilidad, en Nuevas Formulaciones en las ciencias penales, Homenaje a Claus Roxin, Facultad de Derecho y Ciencias Sociales de la Universidad Nacional de Córdoba (Ed. La Lectura y Lerner), Córdoba, 2001.

CoRTÉs de ARABIA, Ana María: Algunos conceptos de la problemática penal aplicables a la actividad curativa, en Nuevas Formulaciones en las ciencias penales, Homenaje a Claus Roxin, Facultad de Derecho y Ciencias Sociales de la Universidad Nacional de Córdoba (Ed. La Lectura y Lerner), Córdoba, 2001.

Demetrio Crespo, Eduardo: Sobre el comienzo de la tentativa en la autoría mediata, en Nuevas Formulaciones en las ciencias penales, Homenaje a Claus Roxin, Facultad de Derecho y Ciencias Sociales de la Universidad Nacional de Córdoba (Ed. La Lectura y Lerner), Córdoba, 2001.

Di Giorgio, Julio César, y Sanmartino, María Lucrecia: ¿Amparo del Estado contra las decisiones de los particulares? Revista de Derecho Penal, Nro. 2001-1, Ed. Rubinzal-Culzoni, Bs. As., 2001.

Donna, Edgardo Alberto: El concepto de autoría y la teoría de los aparatos de poder, en Nuevas Formulaciones en las ciencias penales, Homenaje a Claus Roxin, Facultad de Derecho y Ciencias Sociales de la Universidad Nacional de Córdoba (Ed. La Lectura y Lerner), Córdoba, 2001.

ElHART, Raúl F.: El delito de balance e informe falso y el bien jurídico protegido, Jurisprudencia Argentina, N. ${ }^{\circ}$ 6261, Buenos Aires, 29 de agosto de 2001, pp. 22 y ss.

Buteler, Enrique: Algunas diferencias entre destrucción y acortamiento de la vida en el Código Penal argentino, en Nuevas Formulaciones en las ciencias penales, Homenaje a Claus Roxin, Facultad de Derecho y Ciencias Sociales de la Universidad Nacional de Córdoba (Ed. La Lectura y Lerner), Córdoba, 2001. 
BALCARCE, Fabián I.: La desesperanzadora evolución del tipo culposo en el Derecho comparado, en Nuevas Formulaciones en las ciencias penales, Homenaje a Claus Roxin, Facultad de Derecho y Ciencias Sociales de la Universidad Nacional de Córdoba (Ed. La Lectura y Lerner), Córdoba, 2001.

García Vitor, Enrique: La tesis del dominio del hecho a través de los aparatos organizados de poder, en Nuevas Formulaciones en las ciencias penales, Homenaje a Claus Roxin, Facultad de Derecho y Ciencias Sociales de la Universidad Nacional de Córdoba (Ed. La Lectura y Lerner), Córdoba, 2001.

Lascano, Carlos Julio: Teoría de los aparatos organizados de poder y delitos empresariales, en Nuevas Formulaciones en las ciencias penales, Homenaje a Claus Roxin, Facultad de Derecho y Ciencias Sociales de la Universidad Nacional de Córdoba (Ed. La Lectura y Lerner), Córdoba, 2001.

Lescano, Rolando R. D.: La interpretación en el art. 302, inc. 1, del Código Penal, Jurisprudencia Argentina, N. ${ }^{\circ}$ 6250, 13 de junio de 2001, pp. 22 y ss.

MaIUlini, Federico: Un caso y tres figuras penales: el hurto, la defraudación por infidelidad y la defraudación por omisión de restituir, Doctrina Judicial, Año XVII, N. ${ }^{\circ}$ 39, del 26 de septiembre de 2001, pp. 223 y ss.

Mir Puig, Santiago: Significado y alcance de la imputación objetiva en Derecho Penal, en Nuevas Formulaciones en las ciencias penales, Homenaje a Claus Roxin, Facultad de Derecho y Ciencias Sociales de la Universidad Nacional de Córdoba (Ed. La Lectura y Lerner), Córdoba, 2001.

—: Sobre la punibilidad de la tentativa inidónea en el Código Penal. Revista de Derecho Penal, Nro. 2001-1, Ed. Rubinzal-Culzoni, Bs. As., 2001.

Morin, Daniel Emilio: ¿La bicicleta como vehículo? Reflexiones sobre el tipo de sustracción de vehículos del inciso $6 .^{\circ}$ del artículo 163 del Código Penal, Suplemento de Jurisprudencia Penal, La Ley, Buenos Aires, 5 de octubre de 2001, pp. 4 y ss.

Muñoz Conde, Francisco: La otra cara de Edmund Mezger: su participación en el proyecto de ley sobre "gemeinschaftsfremde»(1940-1944). Revista de Derecho Penal, Nro. 2001-1, Ed. Rubinzal-Culzoni, Bs. As., 2001.

PÉrez BarberÁ, Gabriel E.: Causalidad y determinabilidad, en Nuevas Formulaciones en las ciencias penales, Homenaje a Claus Roxin, Facultad de Derecho y Ciencias Sociales de la Universidad Nacional de Córdoba (Ed. La Lectura y Lerner), Córdoba, 2001.

Pessoa, Nelson R.: Fundamentos de la punición de la tentativa, en Nuevas Formulaciones en las ciencias penales, Homenaje a Claus Roxin, Facultad de Derecho y Ciencias Sociales de la Universidad Nacional de Córdoba (Ed. La Lectura y Lerner), Córdoba, 2001.

Prunotto Laborde, Adolfo: Principio de legalidad. Alcances y precisiones. Revista de Derecho Penal, Nro. 2001-1, Ed. Rubinzal-Culzoni, Bs. As., 2001. 
ReINALDI, Victor F.: Los actos sexuales delictivos en el Código Penal alemán, en Nuevas Formulaciones en las ciencias penales, Homenaje a Claus Roxin, Facultad de Derecho y Ciencias Sociales de la Universidad Nacional de Córdoba (Ed. La Lectura y Lerner), Córdoba, 2001.

Romeo Casabona, Carlos María: Principio de culpabilidad, prevención delictiva y herencia genética, en Nuevas Formulaciones en las ciencias penales, Homenaje a Claus Roxin, Facultad de Derecho y Ciencias Sociales de la Universidad Nacional de Córdoba (Ed. La Lectura y Lerner), Córdoba, 2001.

Roxin, Claus: Injerencia e imputación objetiva, en Nuevas Formulaciones en las ciencias penales, Homenaje a Claus Roxin, Facultad de Derecho y Ciencias Sociales de la Universidad Nacional de Córdoba (Ed. La Lectura y Lerner), Córdoba, 2001.

-: Transformaciones de la teoría de los fines de la pena, en Nuevas Formulaciones en las ciencias penales, Homenaje a Claus Roxin, Facultad de Derecho y Ciencias Sociales de la Universidad Nacional de Córdoba (Ed. La Lectura y Lerner), Córdoba, 2001.

SaAvedra, Heriberto V.: Delitos y faltas electorales, El Derecho, t. 192, Buenos Aires, pp. 789 y ss.

SÁnchez-Ostiz, Pablo: A propósito de la relación entre el encubrimiento y la complicidad en el hecho previo, en Nuevas Formulaciones en las ciencias penales, Homenaje a Claus Roxin, Facultad de Derecho y Ciencias Sociales de la Universidad Nacional de Córdoba (Ed. La Lectura y Lerner), Córdoba, 2001.

Serrano-Piedecasas Fernández: Fundamentación objetiva de la tentativa en el Código Penal español, en Nuevas Formulaciones en las ciencias penales, Homenaje a Claus Roxin, Facultad de Derecho y Ciencias Sociales de la Universidad Nacional de Córdoba (Ed. La Lectura y Lerner), Córdoba, 2001.

Silva Sanchez, Jesús-María: La dimensión temporal del delito y los cambios de «status» jurídico-penal del objeto de la acción, en Nuevas Formulaciones en las ciencias penales, Homenaje a Claus Roxin, Facultad de Derecho y Ciencias Sociales de la Universidad Nacional de Córdoba (Ed. La Lectura y Lerner), Córdoba, 2001.

Spolansky, Norberto Eduardo: Derecho penal, lenguaje y conflictos normativos, en Nuevas Formulaciones en las ciencias penales, Homenaje a Claus Roxin, Facultad de Derecho y Ciencias Sociales de la Universidad Nacional de Córdoba (Ed. La Lectura y Lerner), Córdoba, 2001.

Terradillos Basoco, Juan M.: Culpabilidad y prevención: anotaciones desde el Derecho Penal español, en Nuevas Formulaciones en las ciencias penales, Homenaje a Claus Roxin, Facultad de Derecho y Ciencias Sociales de la Universidad Nacional de Córdoba (Ed. La Lectura y Lerner), Córdoba, 2001. 
Terragni, Marco Antonio: Autoría e intervención de terceros en el delito culposo, en Nuevas Formulaciones en las ciencias penales, Homenaje a Claus Roxin, Facultad de Derecho y Ciencias Sociales de la Universidad Nacional de Córdoba (Ed. La Lectura y Lerner), Córdoba, 2001.

VALdÁguA, María da Conceicao: Figura central, aliciamento e autoría mediata, en Nuevas Formulaciones en las ciencias penales, Homenaje a Claus Roxin, Facultad de Derecho y Ciencias Sociales de la Universidad Nacional de Córdoba (Ed. La Lectura y Lerner), Córdoba, 2001.

VÁsquez, Roberto V.: El uso de documento falso o adulterado, Suplemento de Jurisprudencia Penal, La Ley, Buenos Aires, 7 de septiembre de 2001, pp. 1 y ss.

VERA BARrios, Oscar Tomás: Asociación ilícita, en Nuevas Formulaciones en las ciencias penales, Homenaje a Claus Roxin, Facultad de Derecho y Ciencias Sociales de la Universidad Nacional de Córdoba (Ed. La Lectura y Lerner), Córdoba, 2001.

Vidal Albarracín, Héctor G.: ¿Hasta cuándo subsiste el control aduanero a los fines del contrabando?, Suplemento de Jurisprudencia Penal, La Ley, Buenos Aires, 9 de noviembre de 2001, pp. 16 y ss.

-: Territorio aduanero. Su validez constitucional. Incidencia sobre el delito de contrabando, Suplemento de Jurisprudencia Penal, La Ley, Buenos Aires, 4 de mayo de 2001, pp. 34 y ss.

VIDAL, Humberto S.: El contenido subjetivo del desistimiento voluntario, en Nuevas Formulaciones en las ciencias penales, Homenaje a Claus Roxin, Facultad de Derecho y Ciencias Sociales de la Universidad Nacional de Córdoba (Ed. La Lectura y Lerner), Córdoba, 2001.

Virgolini, Julio, y Silvestroni, Mariano: Unas sentencias discretas. Sobre la discrecionalidad judicial y el Estado de derecho. Revista de Derecho Penal, Nro. 2001-1, Ed. Rubinzal-Culzoni, Bs. As., 2001.

ZafFARONI, Eugenio Raúl: Abolicionismo entre el segundo imperio y la tercera República Francesa, en Nuevas Formulaciones en las ciencias penales, Homenaje a Claus Roxin, Facultad de Derecho y Ciencias Sociales de la Universidad Nacional de Córdoba (Ed. La Lectura y Lerner), Córdoba, 2001.

\section{BRASIL}

(Corresponsal: Prof. Dr. Luiz Regis Prado)

\section{A) Libros}

Barros, Carmen Silvia de Moraes: A individualização da pena na execução penal. São Paulo, RT, 2001. 
Béze, Patricia Mothé Glioche: Concurso formal e crime continuado. Rio de Janeiro, Renovar, 2001.

Bitencourt, Cezar Roberto: Crimes contras as finanças públicas. São Paulo, Saraiva, 2001.

Borges, Fátima Aparecida de Souza: Liberdade provisória. Belo Horizonte, Del Rey, 2001.

BRANDão, Cláudio: Teoria jurídica do crime. Rio de Janeiro, Forense, 2001.

Carvalho, Gisele Mendes de: Aspectos jurídico-penais da eutanásia. São Paulo, IBCCrim, 2001.

CopetTI, André (coord.): Criminalidade moderna e Reformas Penais-Estudos em homenagem ao Professor Luiz Luisi. Porto Alegre, Livraria do Advogado, 2001.

Costa, Paula Bajer Fernandes Martins da: A igualdade no Direito Processual Penal brasileiro. São Paulo, RT, 2001.

D’Avila, Fábio Roberto: Crime culposo e a teoria da imputação objetiva. São Paulo, RT, 2001.

Dotтr, René Ariel: Curso de Direito Penal, Parte Geral. Rio de Janeiro, Forense, 2001.

FerRARI, Eduardo Reale: Medidas de segurança e Direito Penal no Estado Democrático de Direito. São Paulo, RT, 2001.

Franco, Alberto Silva; Stoco, Rui, et alii: Código Penal e sua interpretação jurisprudencial. 7. ed. São Paulo, RT, 2001.

-: Leis penais especiais e sua interpretação jurisprudencial. 7. ed. São Paulo, RT, 2001.

Freitas, Gilberto Passos de; Freitas, Vladimir Passos de: Abuso de autoridade. 9. ed. São Paulo, RT, 2001.

—: Crimes contra a natureza. 7. ed. São Paulo, RT, 2001.

Gomes, Luiz Flávio: Crimes previdenciários. São Paulo, RT, 2001.

—: Erro de tipo e erro de proibição. 5. ed. São Paulo, RT, 2001.

—: Presunção de violência nos crimes sexuais. São Paulo, RT, 2001.

Gomes, Luiz Flávio; BianchinI, Alice: Crimes de responsabilidade fiscal. São Paulo, RT, 2001.

Gomes FiLHo, Antonio Magalhães: A motivação das decisões penais. São Paulo, RT, 2001.

Gonçalves, Victor Eduardo Rios: Crimes hediondos, tóxicos, terrorismo, tortura. São Paulo, Saraiva, 2001.

Grinover, Ada Pellegrini; Gomes Filho, Antonio Magalhães; Fernandes, Antonio Scarance: Recursos no Processo Penal. 3. ed. São Paulo, RT, 2001.

Gullo, Roberto Santiago Ferreira: Direito Penal econômico. Rio de Janeiro, Lumen Juris, 2001. 
Hora, Nilo César Martins Pompílio da: Ação penal: retrospectiva e perspectiva. Rio de Janeiro, Lumen Juris, 2001.

Jesus, Damásio E. DE: Crimes de porte de arma de fogo e assemelhados. 3. ed. São Paulo, Saraiva, 2001.

Lima, Marcellus Polastri: Novas leis especiais criminais. v.1. Rio de Janeiro, Lumen Juris, 2001.

Lisbon, Carolina Cardoso Guimarães: A relação extradicional no Direito brasileiro. Belo Horizonte, Del Rey, 2001.

Machado, Agapito: Juizados Especiais Criminais na Justiça Federal. São Paulo, Saraiva, 2001.

MaIA, Marrielle: Tribunal Penal Internacional. Belo Horizonte, Del Rey, 2001.

Maluly, Jorge Assaf: Denunciação caluniosa. São Paulo, Aide, 2001.

Martins, Jorge Henrique Schaefer: Penas alternativas. Curitiba, Juruá, 2001.

Moura, Maria Thereza Rocha de Assis: Justa causa para a ação penal. São Paulo, RT, 2001.

Nahum, Marco Antonio Rodrigues: Inexigibilidade de conduta diversa. São Paulo, RT, 2001.

Pierangeli, José Henrique: Códigos Penais do Brasil: evolução histórica. 2. ed. São Paulo, RT, 2001.

—: O consentimento do ofendido. 3. ed. São Paulo, RT, 2001.

Pozzer, Benedito Roberto Garcia: Correlação entre acusação e sentença no Processo Penal brasileiro. São Paulo, IBCCrim, 2001.

Prado, Luiz Regis: Crimes contra o ambiente. 2. ed. São Paulo, RT, 2001.

Prado, Luiz Regis (coord.): Responsabilidade penal da pessoa jurídica: em defesa do princípio da imputação penal subjetiva. São Paulo, RT, 2001.

QueIroz, Paulo de Souza: Direito Penal: introdução crítica. São Paulo, Saraiva, 2001.

Rangel, Patrícia Calmon: Abuso sexual. Curitiba, Juruá, 2001.

Rodrigues, Anabela Miranda: Novo olhar sobre a questão penitenciária. São Paulo, RT, 2001.

Rodrigues, João Gaspar: Tóxicos: abordagem crítica da Lei 6.368/76. Campinas, Bookseller, 2001.

SAlomão, Heloisa Estellita: A tutela penal e as obrigações tributárias na Constituição Federal. São Paulo, RT, 2001.

SAnt'Anna, Aline Albuquerque: A nova genética e a tutela penal da integridade física. Rio de Janeiro, Lumen Juris, 2001.

SAntos, Maria Celeste Cordeiro Leite (coord.): Biodireito: ciência da vida, os novos desafios. São Paulo, RT, 2001. 
SHecaIRA, Sergio Salomão (coord.): Estudos criminais em homenagem a Evandro Lins e Silva. Belo Horizonte, Método, 2001.

Scнмidт, Andrei Zenkner: O princípio da legalidade penal no Estado Democrático de Direito. Porto Alegre, Livraria do Advogado, 2001.

SILVA, Cesar Antonio DA: Lavagem de dinheiro: uma nova perspectiva penal. Porto Alegre: Livraria do Advogado, 2001.

StLva, Luciana Caetano DA: Fauna terrestre no Direito Penal brasileiro. Belo Horizonte, Mandamentos, 2001.

Sobrane, Sérgio Turra: Transação penal. São Paulo, Saraiva, 2001.

SouzA, Paulo Vinícius Sporleder DE: A criminalidade genética.São Paulo, RT, 2001.

SuXBERgER, Antonio Henrique Graciano: Responsabilidade penal sucessiva nos crimes de imprensa. Porto Alegre, SAFE, 2001.

TeIXeIRA, João Luís Vieira: Armas de fogo. São Paulo, LTr, 2001.

Welzel, Hans: $O$ novo sistema jurídico-penal. Trad. Luiz Regis Prado. São Paulo, RT, 2001.

Zanatta, Airton: A transação penal e o poder discricionário do Ministério Público. Porto Alegre, SAFE, 2001.

\section{B) Artículos}

AlmeIDA, Gabriel Bertin DE: A crise do conceito tradicional de culpabilidade segundo o Direito Penal contemporâneo. Revista Brasileira de Ciências Criminais. São Paulo, RT, v.35, jul./set.2001.

Amaral, Thiago Bottino Do: Considerações sobre a origem e evolução da ação de habeas corpus. Revista Brasileira de Ciências Criminais. São Paulo, RT, v.35, jul./set.2001.

ARguelho, Silvana Sampaio: A incorporação dos tratados internacionais de proteção dos direitos humanos ao direito brasileiro: a questão da prisão do depositário infiel. Revista Brasileira de Ciências Criminais. São Paulo, RT, v.34, abril/jun.2001.

BARROSO, Luís Roberto; TiBuRCio, Carmen: Algumas questões sobre a extradição no direito brasileiro. Revista dos Tribunais. São Paulo, RT, v.787, maio.2001.

Bastos, João José Caldeira: Crime de omissão de socorro: divergências interpretativas e observações críticas. Revista Brasileira de Ciências Criminais. São Paulo, RT, v.34, abril/jun.2001.

—: Maus-tratos: interpretação do Código Penal e confronto com o delito de tortura. Revista Brasileira de Ciências Criminais. São Paulo, RT, v.35, jul./set.2001. 
Beristain, Antonio: Menores infractores-víctimas ante las Naciones Unidas y el Consejo de Europa. Revista Brasileira de Ciências Criminais. São Paulo, RT, v.34, abril/jun.2001.

—: Protagonismo de las victimas en la ejecución penal (hacia un sistema penitenciario europeo). Revista Brasileira de Ciências Criminais. São Paulo, RT, v.35, jul./set.2001.

CABETte, Eduardo Luiz Santos: O papel do inquérito policial no sistema acusatório - o modelo brasileiro. Revista Brasileira de Ciências Criminais. São Paulo, RT, v.35, jul./set.2001.

Calmon Filho, Petrônio: A investigação criminal na reforma do Código de Processo Penal: agilidade e transparência. Revista Brasileira de Ciências Criminais. São Paulo, RT, v.34, abril/jun.2001.

CARvalHo, Salo: A atual política brasileira anti-drogas: os efeitos do processo eleitoral de 1998. Revista Brasileira de Ciências Criminais. São Paulo, RT, v.34, abril/jun.2001.

CHoukr, Fauzi Hassan: Comentários sobre o anteprojeto de investigação criminal. Revista dos Tribunais. São Paulo, RT, v.789, jul.2001.

Colomer, Juan-Luis Gómez: La instrucción del proceso penal en España y los derechos del imputado. Revista Brasileira de Ciências Criminais. São Paulo, RT, v.34, abril/jun.2001.

Costa, José de Faria: O fenómeno da globalização e o direito penal econômico. Revista Brasileira de Ciências Criminais. São Paulo, RT, v.34, abril/jun.2001.

FAGGIONI, Luiz Roberto Cicogna: O sujeito passivo nos crimes contra a administração pública. Revista Brasileira de Ciências Criminais. São Paulo, RT, v.35, jul./set.2001.

Garcete, Carlos Alberto: O princípio da insignificância. Revista dos Tribunais. São Paulo, RT, v.789, jul.2001.

Gomes, Luiz Flávio: Delito de bagatela: princípios da insignificância e da irrelevância penal. Revista dos Tribunais. São Paulo, RT, v.789, jul.2001.

KARAM, Maria Lúcia: Sobre o ônus da prova na ação penal condenatória. $R e$ vista Brasileira de Ciências Criminais. São Paulo, RT, v.35, jul./set.2001.

Lima, Wanderson Marcello Moreira DE: Presunção de inocência. Revista dos Tribunais. São Paulo, RT, v.786, abr.2001.

Marcão, Renato Flávio; Marcon, Bruno: Rediscutindo os fins da pena. Revista dos Tribunais. São Paulo, RT, v.786, abr.2001.

Mendroni, Marcelo Batlouni: Tópicos essenciais da lavagem de dinheiro. Revista dos Tribunais. São Paulo, RT, v.787, maio.2001.

Mossin, Heráclito A.: Crime de tortura. Revista dos Tribunais. São Paulo, RT, v.786, abr.2001. 
NAves, Maurílio Cardoso: A pena substitutiva de prestação pecuniária: hipóteses de aplicação e critérios de fixação. Revista dos Tribunais. São Paulo, RT, v.787, maio.2001.

Nores, José I. Cafferata: La eficacia de la investigación penal en el Estado de Derecho. Revista Brasileira de Ciências Criminais. São Paulo, RT, v.35, jul./set.2001.

PRADO, Luiz Regis: Os novos crimes contra as finanças públicas. Revista dos Tribunais. São Paulo, RT, v.786, abr.2001.

Roxin, Claus: Sobre a fundamentação político-criminal do sistema jurídicopenal. Revista Brasileira de Ciências Criminais. São Paulo, RT, v.35, jul./set.2001.

SANTANA, Selma Pereira DE: Contornos de uma doutrina teleológica-racional do crime. Revista Brasileira de Ciências Criminais. São Paulo, RT, v.35, jul./set.2001.

Silva SÁnchez, Jesús María: La consideración del comportamiento de la víctima en la teoría jurídica del delito. Observaciones doctrinales y jurisprudenciales sobre la «victimo-dogmática». Revista Brasileira de Ciências Criminais. São Paulo, RT, v.34, abril/jun.2001.

SiqueIRa Jr., Paulo Hamilton: Interrogatório a distância-on line. Revista dos Tribunais. São Paulo, RT, v.788, jun.2001.

Velloso, Carlos: A justiça e seu problema maior: a lentidão. Justiça, Ministério Público e polícia: a tripeça em que se assenta a segurança pública. O juizado de instrução. Revista dos Tribunais. São Paulo, RT, v.788, jun.2001.

VIDAL, Hélvio Simões: Causalidade, ciência e experiência em matéria penal. Revista dos Tribunais. São Paulo, RT, v.788, jun.2001.

\section{GENTROAMÉRICA}

(Corresponsal: D. Álvaro Burgos)

\subsection{COSTA RICA}

Castillo, Francisco: El error de prohibición. Editorial Juritexto, 164 pp., 2001.

Llobet, Javier: Proceso Penal en la Jurisprudencia. Editorial Jurídica Continental, 2 Tomos, 997 pp., 2001.

SALINAS, Edwin: El procedimiento abreviado: mitos y realidades. Editorial Mundo Gráfico, 135 pp., 2001.

VV. AA.: Garantías constitucionales, prueba ilítica y la transición al nuevo proceso penal. Editorial Investigaciones Jurídicas S. A., 455 pp., 2001. 


\subsection{GUATEMALA}

ANíbal, Héctor: Derecho Penal Guatemalteco. Editorial Lerena, 777 pp., 2000. FigueroA, Raúl: Código Procesal Penal. Editorial Lerena, 258 pp., 2000.

\subsection{NICARAGUA}

Cuarezma, Sergio/Howed, Mario: Derecho Penal, Criminología y Derecho Procesal Penal. Editorial Hispamer, 660 pp., 2000.

\section{CHILE}

(Corresponsal: D. Juan Cárcamo Olmos)

\section{A) Revistas}

La Revista de Derecho. Derecho-Sociedad-Cultura. Universidad Central de Chile. Facultad de Ciencias Jurídicas y Sociales. Año VI Nro. 1. 2 da. época. Enero-Diciembre 2.000. Santiago de Chile, año 2.000.

Carcamo Olmos, Juan Carlos: «Análisis de la nueva ley sobre delitos de conducta sexual desde el punto de vista del Derecho penal sustantivo».

Revista de Derecho, Criminología y Ciencias penales. Facultad de Derecho. Instituto de Criminología. Universidad San Sebastián. Año II Nro. 2. 2.000 Concepción-Chile.

Barbero Santos, Marino: «Estado Constitucional de Derecho y Sistema Penal». SAEZ MuÑoz, Rodolfo: «Criminalística, peritos y peritajes».

Romeo Casabona, Carlos María: «Bienes jurídicos implicados en la donación».

Kunsemuller Loebenfelder, Carlos: «Principio de culpabilidad-culpabilidad. Notas sobre su evolución y "crisis"».

Carcamo Olmos, Juan Carlos: «Breves reflexiones de Política Criminal en el Derecho penal económico».

Blanco Lozano, Carlos: «El principio de violencia en el Derecho penal». Silva Siulva, Hernan: «Generalidades sobre la reforma procesal Penal».

Actualidad Jurídica. Revista. Facultad de Derecho. Universidad del Desarrollo. Año I. Nro. 1. Enero 2.000. Santiago de Chile.

RoJas S., Mario: «El principio territorial, juzgamiento penal y estricta jurisdiccionalidad».

Actualidad Jurídica. Revista. Facultad de Derecho. Universidad del Desarrollo. Año I. Nro. 2. Julio 2.000. Santiago de Chile. 
Rojas S., Mario: «Corte Penal Internacional y soberania universal».

Revista de Derecho. Consejo de Defensa del Estado. Año I. Santiago de Chile, Julio 2.000. Nro. 1.

Szczaranski Cerda, Clara, y Muñoz Ortuzar, Maria Teresa: «De tempore delicti».

Revista de Derecho. Consejo de Defensa del Estado. Año I. Santiago de Chile, Diciembre 2.000. Nro. 2.

Rivacoba Y Rivacoba, Manuel DE: «Una sentencia de desafuero Trascendental y famosa».

RuIz Pulido, Guillermo: «Una sentencia insólita».

Revista de Derecho. Universidad Católica de Valparaíso. XX - 1.999. Ediciones Universitarias de Valparaíso.

Solari, Tito: «Presupuestos teóricos del ministerio publico».

\section{COLOMBIA}

(Corresponsal: Prof. Dr. Alfonso Cadavid Quintero)

\section{A) Libros}

Agudelo Betancur, Nódier: Embriaguez y responsabilidad penal.

Agudelo Betancur, Nódier: La defensa putativa en el nuevo Código penal.

Albrecht, Hans-Jörg: Criminalidad transnacional, comercio de narcóticos y lavado de dinero (Trad. O. J. Guerreo Peralta).

Amaya Velosa, Campo E.: El drama de las cárceles en Colombia.

Arboleda Vallejo, Mario, y Ruiz Salazar, José A.: Manual de Derecho penal.

Arboleda Vallejo, Mario, y Ruiz Salazar, José A.: Manual de Derecho penal, tomo II, parte especial. Los delitos en particular. Ed. Leyer.

Arboleda Vallejo, Mario, y Ruiz Salazar, José A.: Nuevo Código penal comentado (ley 599 de 2000).

Bacigalupo, Enrique, et al.: Monografías penales.

Botero Bernal, José F.: De la manipulación genética en el nuevo Código penal colombiano.

Camargo, Pedro P.: La extradición.

CANcio Meliá, Manuel: Conducta de la víctima e imputación objetiva en Derecho penal.

CAncio Meliá, Manuel, et al.: XXIII Jornadas internacionales de Derecho penal (U. Externado de Colombia).

CARranza Piña, Jorge E.: La inculpabilidad y la inimputabilidad social en el Código penal. 
CARranza PiÑa, Jorge E.: El sujeto, el poder y las penas en el nuevo Código penal. CARreño, Bernardo-Sierra, Nubia: Hacia una filosofía del Derecho penal en Latinoamérica.

CERón Erazo, Leonardo E.: La víctima, el protagonista desplazado del conflicto penal.

Córdoba Angulo, Miguel: La tentativa (2. ${ }^{a}$ reimpresión).

Córdoba Triviño, Jaime: Derecho penal internacional.

CORTÉs PÉREz, María A.: El menor infractor.

Cuello Vengal, Unaldo D.: Teoría del delito de patrimonio económico en el nuevo Código penal colombiano.

CHERIF BAssiouni et al.: La Corte penal internacional.

Escobar LóPEz, Edgar: Estupefacientes, delitos y contravenciones.

FIERRO-MÉndez, Heliodoro: La responsabilidad penal en los contratos administrativos.

Gómez López, Jesús Orlando: Tratado de Derecho penal, tomos I y II.

Gómez Pavajeau, Carlos A.: Constitución, derechos fundamentales y dogmática penal (1. reimp.)

González Monguí, Pablo E.: Derecho penal en las relaciones laborales, públicas y privadas.

Herrera, Gladis: La manipulación genética a la luz del Derecho penal.

Higuita Rivera, Lina M. ${ }^{a}$ : La responsabilidad penal del servidor público.

JAKoBS, Gunther: Injerencia y Derecho penal (Trad. M. Cancio M.).

Jiménez Moreno, Juan G.: Aproximación a la manipulación genética.

León Moncaleano, William F.: De la comunicación a la informática judicial penal bancaria.

López Morales, Jairo: Nuevo Código penal, tomos I, II, III.

Mojica Monsalvo, Danilo: Responsabilidad penal del ebrio.

Monroy Cabra, Marco G., y Navarro del Valle, Hermes: Desaparición forzada de personas.

Pabón Parra, Pedro A.: Comentarios al nuevo Código penal sustancial.

Pabón Parra, Pedro A.: Delitos contra la familia conforme al nuevo Código penal.

Palacio, Marisol: Contribuciones de la victimología al sistema penal.

Parra Gutiérrez, William, y Parra Mejía, Andrea: Estupefacientes y adicciones.

PeÑa Velásouez, Edgard: Comentarios al Código penal militar.

Perdomo Torres, Jorge F.: La problemática de la posición de garante en los delitos de comisión por omisión.

Pérez Pinzón, Álvaro O.: Curso de criminología (6. ${ }^{\mathrm{a}}$ ed.). 
Posada Maya, Ricardo, y Hernández Beltrán, Harold: El sistema de individualización judicial de la pena en el derecho penal colombiano.

Urbano Martínez, José J.: La legitimidad del Derecho penal.

Vargas Vargas, Pedro P.: «Derecho Penal General», en Nuevo Código penal 2001.

Villanueva Mesa, Javier Antonio, y Zuleta Cano, José Abad: Jurisdicción penal internacional.

\section{B) Revistas}

Revista de Derecho penal, núm. 22 (diciembre 2000-enero 2001)

CAmargo, Pedro P.: «El delito de trata y tráfico de personas».

Mezger, E.: «La acción».

Pabón Parra, Pedro A.: «El delito de daño en la legislación colombiana. Evolución normativa y análisis dogmático».

PRADEL, Jean: «Los sistemas penales frente al reto del crimen organizado».

ZAFFARONI, Eugenio: «En defensa del Derecho penal liberal».

\section{Revista de Derecho penal, núm. 23 (febrero-marzo 2001)}

Botero Bernal, José F.: «Protección jurídico-penal a la vida humana»Aproximación crítica a las conductas punibles vertidas en el Título I del Libro II del Nuevo Código Penal-.

Camargo, Pedro P.: «La pena de muerte y la cadena perpetua».

Dorado Montero, Pedro: «Del Derecho penal represivo al preventivo».

PABón P., Pedro A.: «El delito de extorsión. La acción, hipótesis de concurso y diferenciación».

Ramino Ramos, M. H., y KÜNSEMÜlleRL, C.: «Los sistemas penales frente al reto del crimen organizado».

RodRíGuez, Gabriela: «Tráfico internacional de personas desde la perspectiva de los derechos humanos».

Zamora A., Martha L.: «Investigación judicial del tráfico de personas».

\section{Revista de Derecho penal, núm. 24 (abril-mayo 2001)}

Botero Bernal, José F.: «Bosquejo crítico al Título V del Libro II del nuevo Código Penal: "Delitos contra la integridad moral"».

CAmargo, Pedro P.: «La doble instancia y la non reformatio in pejus».

JimÉneZ Moreno, Juan G.: «¿Se justifica la desjudicialización de la Fiscalía?». MERKEL., A.: «Derecho penal». 
Molina A., Carlos M.: «Principio de restablecimiento y reparación del Derecho».

Pabón P., Pedro A.: «El bien jurídico de la administración de justicia en el nuevo Código Penal».

RoBinson, Mary: "Informe de la ONU sobre derechos humanos en Colombia».

Villanueva, Gustavo A.: «Control de legalidad en las medidas de aseguramiento».

\section{Revista de Derecho penal núm. 25 (junio-julio 2001)}

El estado peligroso:

- JimÉnEZ DE AsúA, Luis: «Nueva fórmula para el tratamiento penal y preventivo».

- Soler, Sebastián: «Exposición y crítica de la teoría del estado peligroso".

Botero Bernal, Andrés : «La teoría unificadora dialéctica de Roxin a la luz de Beccaria».

CAmargo, Pedro P.: «El nuevo Código penal y la represión de los delitos contra el Derecho Internacional Humanitario (delicta juris genium)».

SAAVEDRA RoJAS, Edgar: "El principio de legalidad desde la perspectiva del Derecho internacional de los derechos humanos y del Derecho humanitario».

FIERRO-MÉndez, Heliodoro: «La jurisprudencia como fundamento de las decisiones judiciales en Colombia».

Figueroa Morantes, Jorge E.: «La dinámica probatoria en el esquema del proceso penal colombiano».

Jiménez MoReno, Juan Guillermo: «La resolución de apertura de instrucción y la vinculación de autores y partícipes en el nuevo Código de procedimiento penal».

Molina A., Carlos M.: «Principio de igualdad: Noción y contenido. Desarrollos jurisprudenciales».

Pabón P., Pedro Alfonso: «El abuso de confianza».

ZAFFARONI, Eugenio: «El racismo como estructura discursiva contra los derechos humanos».

\section{Revista de Derecho penal núm. 26 (agosto-septiembre 2001)}

CAmargo, Pedro P.: «El habeas hábeas en el nuevo Código Procesal penal».

CAnçado Trindade, Antonio A.: «El nuevo reglamento de la Corte Interamericana de derechos humanos (2000): La emancipación del ser humano como sujeto de Derecho internacional de los derechos humanos». 
EscoBAR LóPEz, Edgar: «Clonación y manipulación genética».

FLorí́n, Eugenio: «De las penas y de las instituciones afines».

GAITÁN M., Bernardo: «La extradición de hecho».

López P., Gerardo: «Breve comentario a las normas rectoras del Código de procedimiento penal».

Pabón Parra, Pedro A.: «La familia como bien jurídico protegido en el nuevo Código Penal».

PARAYRE, Sonia: «La desaparición forzada de personas como violación continuada de los derechos humanos y su incidencia en la determinación de la competencia ratione temporis de la Corte Interamericana de derechos humanos».

\section{Nuevo Foro Penal 63}

CADAVID Quintero, Alfonso: “Habeas hábeas” y prevaricación judicial».

CAlle C., Armando: «Bases para una fundamentación político-constitucional del debido proceso».

Escobar MeJía, J. Guillermo: «Las banderas en el aire, Homenaje a Jesús M. ${ }^{a}$ Valle».

Fernando Reyes C., José: «Término de la celebración de la audiencia pública y libertad provisional».

Londoño B., Hernando, y VARGas R., Alicia E.: «El indígena ante el Derecho penal colombiano».

LondoÑo J., Hernando: «Cómo se atenta contra la libertad en el proceso penal».

VARGAS, Álvaro: «Construcción, de(s)construcción y reconstrucción del proceso penal de la modernidad».

Velásouez V., Fernando: «La teoría de la conducta punible en el nuevo Código penal».

\section{PERÚ}

(Corresponsal: Prof. Dr. D. José Urquizo Olaechea)

\section{A) Libros}

Abanto Vásouez, Manuel: Los delitos contra la Administración Pública en el Código Penal peruano, Palestra editores, Lima, 2001.

Bramont Arias, Luis, y Bramont Arias-Torres, Luis Alberto: Código Penal anotado, reimpresión de la $3^{\text {a }}$ ed., Editorial San Marcos, Lima, 2001. 
CARo Coria, Dino Carlos: Código Penal y Legislación Complementaria, Gráfica Horizonte, Lima, 2001, ps. 474.

Castillo Alva, José Luis: Las Consecuencias Jurídicas-Económicas del Delito, Ed. Idemsa, Lima, 2001. ps. 321.

—: Falsedad Documental, Jurista Editores, Lima, 2001, ps. 243.

—: La violación sexual en el Derecho Penal, Jurista Editores, Lima, 2001, ps. 150.

Comisión Andina de JuRistas: La Corte Penal Internacional y los países andinos, Comisión Andina de Juristas, Lima, 2001.

Frisancho Aparicio, Manuel: Delitos contra la administración de Justicia, Jurista Editores, Lima, 2001.

Hurtado Pozo, José: Derecho Penal y Discriminación de la Mujer-Anuario de Derecho Penal 1999-2000, Fondo Editorial de Universidad Católica del Perú, Lima, 2001, ps. 506.

JaÉN VAllejo, Manuel: Tendencias Actuales de la Jurisprudencia Penal Española-Derecho procesal penal y derecho penal sustantivo, Ed. Gráfica Horizonte, Lima, 2001, ps. 234.

Paredes Infanzón, Jelio: Delitos contra la Fe Pública, Jusistas Editores, Lima, 2001.

Peña Cabrera, Raúl A.: Practica Forense Penal, Jurista Editores, Lima, 2001.

QuisPe FARfÁn, Fany Soledad: El Derecho a la Presunción de Inocencia, Palestra Editores, Lima, 2001, ps 166.

Rojas Vargas, Fidel: Delitos contra la Administración Público, $2^{\circ}$ edición, Ed. Grijley, Lima, 2001, ps 966.

Rojas Vargas, Fidel, y Infantes Vargas, Alberto: Código Penal. Diez años de Jurisprudencia sistematizada, Ed. Idemsa, Lima, 2001

Villa Stein, Javier: Derecho Penal, Parte General, $2^{\circ}$ edición, Ed. San Marcos, Lima, 2001, ps 617.

Villavicencio Terreros, Felipe: Código Penal Comentado, Ed. Grijley, Lima, 2001, ps. 917.

\section{B) Revistas}

\section{Cathedra}

N. ${ }^{\circ} 07 / 2000$ (diciembre)

Coz Ramos, Ernesto: Comentarios en relación al delito de lavado de dinero en la legislación peruana, p. 81.

BотткE, Wilfried: Sexualidad y delito: las víctimas de los delitos sexuales. 


\section{Derecho y Sociedad}

\section{N. ${ }^{\circ} 16 / 2001$}

Lamas Puccio, Luis: Corrupción de Funcionarios y Lavado de Dinero, p. 89.

Grupo de Investigación de Derecho y Sociedad: La Protección Penal del Lavado de Dinero en el Perú, p. 97.

Rodríguez Delgado: Problemática Penal del Honor y de las Libertades de Información de Expresión ¡Libertad de Información o Libertinaje Informativo!, p. 113.

\section{Gaceta Jurídica-Actualidad Jurídica.}

\section{N. ${ }^{\circ} 86 / 2001$}

SAN MARTín CAStro, Cesar Eugenio: Las medidas limitativas de derecho en la investigación preliminar del delito.

Bramont-Arias Torres, Luis Miguel: El delito de Prevaricato.

\section{N. ${ }^{\circ} 87 / 2001$}

Reyna Alfaro, Luis Miguel: Aproximación al derecho penal económico-estereotipo, desigualdad, poder económico e impunidad.

Bramont-Arias Torres, Luis Miguel: El delito de proxenetismo.

\section{$\mathbf{N} .^{\circ} \mathbf{8 8 / 2 0 0 1}$}

García Cantizano, María del Carmen: Algunas consideraciones en torno al delito de tráfico de influencias.

\section{N. ${ }^{\circ} 89 / 2001$}

Castillo Alva, José Luis: El delito de corrupción de magistrados, árbitro, fiscal, miembro del Tribunal Administrativo o de cualquier otro análogo.

Reyna Alfaro, Luis Miguel: Bien Jurídico en el delito informático.

García Cantizano, María del Carmen: El delito de difamación y la aplicación de exceptio veritatis.

\section{$\mathbf{N} .^{\circ}$ 90/2001}

VÁsouez VÁsouez, Mario: Abstención del ejercicio de la acción penal. El principio de Oportunidad.

\section{N. ${ }^{\circ} \mathbf{9 1 / 2 0 0 1}$}

ZuÑIgA RodRíGuEZ, Laura: Los delitos societarios: entre la transformación del Derecho Penal y del Derecho de Sociedades.

Bramont-Arias Torres, Luis Miguel: Actos contra el pudor, exhibiciones y publicaciones obscenas y pornografía infantil. 


\section{Normas Legales-Revista Jurídica del Perú}

N. ${ }^{\circ} 19 / 2001$

Gómez Benavidez, Percy: Leyes anticorrupción en la legislación peruana.

\section{N. ${ }^{\circ} \mathbf{2 0 / 2 0 0 1}$}

NuñEz PAz, Miguel Angel (España): Reflexiones sobre criminología y control social sobre el delito.

Hess Araya, Christhian (Costa Rica): Regulaciones y proyectos sobre delitos informáticos en Costa Rica.

Chacón Corado, Mauro (Guatemala): Las excepciones en el Código Procesal Penal.

N. ${ }^{\circ} 21 / 2001$

Reyna Alfaro, Luis Miguel: El Bien Jurídico en el delito informático.

N. ${ }^{\circ} 23 / 2001$

Chacón Corado, Mauro (Guatemala): La acción civil en el proceso penal.

\section{N. ${ }^{\circ} 25 / 2001$}

Reyna Alfaro, Luis Miguel: El programa penal económico de la Constitución.

Ruiz Rodríguez, Luis Ramón: El delito publicitario en el Código penal español.

Revista Peruana de Doctrina y Jurisprudencia Penal

N. ${ }^{\circ} 2 / 2001$

\section{Notas necrológicas}

Polaino Navarrete, Miguel: Semblanza personal y universitaria de Manuel Rivacoba y Rivacoba (1925 - 2000). In memoriam.

SÁnchez-Ostiz, Pablo: Profesor Arthur Kaufmann (1923 - 2001), in memoriam.

\section{Doctrina}

AlCÁCER GuIRAo, Rafael: ¿Protección de bienes jurídicos o protección de la vigencia de las normas? Consideraciones sobre el destinatario de las normas.

CoRnAcCHIA, Luigi: Causa ad excludendum: la problemática de la interrupción del curso causal.

Marinucchi, Giorgio, y DolcinI, Emilio: Derecho penal «mínimo» y nuevas formas de criminalidad.

FeliP i SABORIt, David: El objeto de conocimiento de la antijuricidad.

HRUSCHKA, Joachim: La conducta de la víctima como clave para un sistema de los delitos patrimoniales que llevan consigo sustracción. 
JAKOBS, Gunter: La denominada actio libera in causa.

KINDHÄUSER, Urs: La estafa por medio de computadoras, ¿una estafa?

Mazuelos Coello, Julio F.: los delitos informáticos: una aproximación del Código Penal peruano.

Mưssig, Bernd: Desmaterialización del bien jurídico y de la política criminal.

PASTOR MuÑoz, Nuria: Estafa y negocio ilícito. Algunas consideraciones a propósito de la STS de 13 de mayo de 1997.

Peñaranda Ramos, Enrique: Función de la pena y sistema del delito desde una orientación preventiva del derecho penal.

Perdomo Torres, Jorge Fernando: Algunas consideraciones dogmáticas sobre la regulación de la comisión por omisión en el nuevo Código Penal colombiano.

Polaino Navarrete, Miguel, y Polaino-Orts, Miguel: ¿Medidas de Seguridad «inocuizadoras» para delincuentes peligrosos? Reflexiones sobre su discutida constitucionalidad y sobre el fundamento y clases de las medidas de seguridad.

RagüES I VALLÈs, Ramón: Tres grandes dilemas de la dogmática penal del siglo XXI.

Stlva Sánchez, Jesús María: Libertad de terapia e imprudencia médica.

ToRío LóPEz, Ángel: Relación de casualidad. Indicaciones para una actualización.

\section{Themis}

\section{N. ${ }^{\circ} 42 / 2001$}

Reaño Pescheira, José: La problemática del Bien Jurídico protegido en el delito de insider trading y técnica de protección, p. 337.

\section{URUGUAY}

(Corresponsal: D. Diego Silva Forné)

\section{A) Libros}

Авоso, Gustavo Eduardo: El delito de defraudación por administración infiel, Editorial B de F, Montevideo-Buenos Aires, 2001, 236 pp.

AA. VV.: La ebriedad en la jurisprudencia penal. Homenaje a Luis Piñeiro Chain, Carlos Alvarez editor, Montevideo, 2001, 120 pp.

DuARTE NoseI, Eduardo: Ley de seguridad ciudadana (Ley $N^{\circ}$ 16.707, modificativas y concordantes). Enfoque doctrinario, Fundación de Cultura Universitaria, Montevideo, 2001, $140 \mathrm{pp}$. 
GreIf, Jaime: Código del Proceso Penal de la República Oriental del Uruguay, anotado y concordado, Fundación de Cultura Universitaria, Montevideo, $152 \mathrm{pp}$.

JAÉN VALLEJo, Manuel: La justicia penal ante los nuevos retos de la actualidad, Fundación de Cultura Universitaria, Montevideo, 2001, 144 pp.

Langón Cuñarro, Miguel: Curso de Derecho Penal y Procesal Penal, tomos II y III, Ed. «Del Foro», Montevideo, 2001, pp. 334 y 284 resp.

Mirabal Bentos, Gustavo: Vademecum de práctica penal y ciencias auxiliares, tomo I, Amalio Fernández editor, Montevideo, 2001624 pp.

Muñoz Conde, Francisco: Introducción al Derecho Penal, $2^{\mathrm{a}}$ ed., Editorial B de F, Montevideo-Buenos Aires, 2001, 324 pp.

Rocco, Arturo: El objeto del delito y de la tutela jurídico-penal (trad. de Gerónimo Seminara), Editorial B de F, Montevideo-Buenos Aires, 2001, 684 pp.

URIOSte BRAgA, Fernando: Responsabilidad internacional de los Estados en los derechos humanos, Editorial B de F, Montevideo-Buenos Aires, 2001, 236 pp.

VIEgA Rodríguez, M ${ }^{\mathrm{a}}$ José: «Un nuevo desafío jurídico: los delitos informáticos», en AA. VV.: Derecho informático, Fundación de Cultura Universitaria, Montevideo, 2001, pp. 185 a 203.

Vieira, Manuel A., y García Altolaguirre, Carlos: Extradición, Fundación de Cultura Universitaria, Montevideo, 2001, 448 pp.

Welzel, Hans: El nuevo sistema del Derecho penal (trad. de José Cerezo Mir), Editorial B de F, Montevideo-Buenos Aires, 2001, 201 pp.

\section{B) Revistas}

\section{Cuadernos de la Facultad de Derecho de la Universidad de la República}

\section{$3^{\mathrm{a}}$ serie, $\mathbf{N}^{\mathrm{o}} 6$}

Gros EsPIELL, Héctor: Responsabilidad del Estado y responsabilidad penal internacional en la protección internacional de los Derechos Humanos, pp. 11-21.

Urioste Braga, Fernando: Responsabilidad penal de los individuos, pp. 45-53.

-: Los crímenes internacionales de Estado y los Derechos Humanos, pp. 61-70.

WINTER, Gabriel: La prohibición de la tortura, pp. 215-226.

Herramientas. Revista interdisciplinaria sobre temas de justicia juvenil N. ${ }^{\circ} 2$

BERRo, Graciela: Internación con medidas de seguridad, pp. 75-81.

CoHEn, Jorge: Las teorías filosóficas como fundamento del sistema penal juvenil, pp. 82-89. 
Ghione, Alicia; Salsamendi, Javier, y Pedernera, Luis: Trazos críticos para la contención del castigo en niños y adolescentes, pp. 100-115.

Hillman, Alejandra, y Gudiño, María: Una puerta abierta a la mediación penal, pp. 16-22.

INTROINI, Mercedes: Mediación y niños infractores, pp. 116-123.

MaXera, Rita: La nueva Ley Orgánica de la responsabilidad penal de los menores de España, pp. 23-35.

ORTE, Carmen: Conductas antisociales, conflictos escolares y consumo de drogas, pp. 36-54.

Silva Balerio, Diego, y Rosich, Martín: La educación social y el control de los adolescentes en conflicto con la ley penal (I), pp. 124-139.

Solís, Gabriela: Sistema penal y sociedad civil. La responsabilidad de la sociedad frente a la cárcel, pp. 55-68.

VIÑAR, Marcelo: Desamparo, minoridad abandonada e infractora y psicoanálisis, pp. 140-165.

\section{Revista de Derecho Penal}

\section{N. ${ }^{\circ} 12$}

Bustos Ramírez, Juan; Buteler, José A., y Muñoz Conde, Francisco: Hacia una construcción latinoamericana de la culpabilidad. Conferencia plenaria, XI Congreso Latinoamericano y III Iberoamericano de Derecho Penal y Criminología, pp. 163-181.

Camaño Viera, Diego: Ley de urgencia y Derecho Penal de la emergencia, pp. 9-17.

Conujo, William: La selectividad de la pena en el Derecho Penal uruguayo, pp. 195-201.

FuENTE, Javier DE LA: El dolo eventual y su diferencia con la culpa, pp. 103-122.

DonnÁngelo, Pablo: Algunas conclusiones del último Congreso Iberoamericano de Derecho Penal y Criminología. ¿Qué nos dejó el evento?, pp. 183-192.

Herzog, Felix: Algunos riesgos del Derecho Penal del riesgo, pp. 203-206.

Laurenzo Copello, Patricia: Algunas reflexiones críticas sobre la tradicional división tripartita del dolo, pp. 123-134.

Malet VÁzouez, Mariana: El agente provocador, pp. 19-46.

Pesce Lavaggi, Eduardo A.: Causales de justificación en la Ley N. ${ }^{\circ}$ 17.243, pp. 47-64.

-: Criminalización de los delitos culposos: despenalización real, pp. 135-142.

Petito SAcco, José A.: Modificaciones en materia de agravantes. El deber de informar, pp. 65-74.

Puig, Gustavo: El incremento de la punición en la legislación uruguaya, pp. $75-77$. 
RivacoBa y RivacoBa, Manuel: La reforma penal de la Ilustración, pp. 504-518. —: Recapitulación y despedida de un penalista, pp. 519-524.

Salom Falcón, Cecilia: El alcohol y la legislación de urgencia, pp. 79-86.

SiLVA Forné, Diego: Juegos de azar, tutela penal de la propiedad y delito de juego de la mosqueta, pp. 87-102.

Sotomayor Acosta, Juan O., y Gallego García, Gloria: El dolo eventual en el Código Penal colombiano: entre limitaciones dogmáticas y exigencias político-criminales, pp. 143-160.

\section{Revista de la Facultad de Derecho de la Universidad de la República}

N. ${ }^{\circ} 17$

Alvarez, Jorge W.: Inmoralidad de la prisión preventiva, pp. 199-200.

LANGÓN CuÑARro, Miguel: La teoría de la vergüenza reintegrativa de John Braithwaite, pp. 63-67.

URIOSTE, Fernando: Jurisdicción penal internacional, pp. 79-84.

\section{Revista Uruguaya de Derecho Procesal}

\section{N. ${ }^{\circ} 2001 / 2$}

STIPANICIC, Emma, y Valentín, Gabriel: La clausura de los procesos penales en la Ley N. ${ }^{\circ} 17.272$, pp. 177-188.

\section{VENEZUELA}

(Corresponsal: Prof. Dr. D. Carlos S. Bello Rengifo)

Arismend, Luis Miguel: Análisis práctico de la Ley Penal del Ambiente Venezolana: tipos penales. Editorial Arismeca, 1999.

ARrAIz, Evelinda, y otros: Victimización de la mujer embarazada producto de una violación. Sin notas tipográficas.

Arteaga Sánchez, Alberto: Derecho Penal Venezolano. McGraw-Hill, 2001.

Borges, Carmen Luisa: Principios penales en el Código Orgánico Tributario. Universidad Central de Venezuela, 1999.

Código Penal de Venezuela Comentado: Tomos VI, VII, VIII, Instituto de Ciencias Penales y Criminológicas, Universidad Central de Venezuela, 1999.

Díaz Chacón, Feddy José: Doctrina Penal del Tribunal Supremo de Justicia. Máximas y extractos. Volúmenes 1, 2, 3, y 4. Livrosca, 2000.

Estudios de Derecho en Homenaje a Fernando Pérez-Llantada: S. J. Universidad Católica Andrés Bello, 2000.

Gil CASTILLO, Roberto: La protección penal del agua. Un análisis de la Ley Penal del Ambiente, Livrosca, 2000. 
Gómez GRILLO, Elio: Apunte sobre la delincuencia y la cárcel en la literatura venezolana. Monte Avila Editores Latinoamericana, 2000.

Jurispenal. Justicia penal. Universidad Central de Venezuela, 2000.

Los Derechos Humanos y la Agenda del Tercer Milenio: XXV Jornadas «J. M. Domínguez Escovar». Tribuna Jurídica de Venezuela. Colegio de Abogados del Estado Lara.

Maldonado, Pedro/Gaviria, José: Drogas. Fondo Editorial de la Universidad Santa María, 2000.

Marcano López, Marcell: El delito de lesiones, Livrosca, 2001.

MARTínez, Moira: Condiciones para la aplicación del sistema penal de responsabilidad previsto en la LOPNA, Universidad Central de Venezuela, 2001.

Modolell González, Juan Luis: Bases fundamentales de la teoría de la imputación objetiva. Livrosca, 2001.

Pérez Chiriboga, Belén: Código Penal de Venezuela. Editorial Jurídica Venezolana, 2000.

Piña Loaiza, Rafael: Dictámenes Jurisprudenciales del Tribunal Supremo de Justicia, Sala de Casación Penal. Editorial Buchivacoa. Año 1, Vol. 1 (enero 2000) y Vol. 2 (febrero 2000).

PRIMER AÑo DE VIGENCIA DE LA LOPNA: Segundas Jornadas sobre la Ley Orgánica de Protección del Niño y del Adolescente, Universidad Católica Andrés Bello, 2001.

SÁnCHEZ, María Guadalupe: Consideraciones generales a la Ley Orgánica para la protección del Niño y del Adolescente, con especial referencia a la responsabilidad penal y la violencia doméstica. Editorial Buchivacoa, 2000.

TAMAYO RodríGUEz, José Luis: Intervenciones telefónicas y grabaciones ilícitas. Escritorio Jurídico Tamayo-Tamayo, 1999.

VilLalba, Carlos: Delito e insurgencia. Universidad Central de Venezuela, 2001. 